\title{
Statistical properties of solar wind discontinuities, intermittent turbulence, and rapid emergence of non-Gaussian distributions
}

\author{
A. Greco*, W. H. Matthaeus ${ }^{\dagger}$, S. Servidio*, P. Dmitruk**, M. Wan ${ }^{\dagger}$, S. Oughton ${ }^{\ddagger}$ and \\ P. Chuychai ${ }^{\S}$ \\ *Dipartimento di Fisica, Universita' della Calabria, Ponte P. Bucci Cubo 31C, Rende, CS 87036, Italy \\ ${ }^{\dagger}$ Bartol Research Institute and Department of Physics \& Astronomy, University of Delaware, Newark DE 19716 \\ ${ }^{* *}$ Departimento de Física (FCEN-UBA), Buenos Aires, Argentina \\ ${ }^{\ddagger}$ Department of Mathematics, Univerrsity of Waikato, Hamilton, $N Z$ \\ ${ }^{\S}$ School of Science Mae Fah Luang University, Chiang Rai, Thailand
}

\begin{abstract}
.
Recent studies have compared properties of the magnetic field in simulations of Hall MHD turbulence with spacecraft data, focusing on methods used to identify classical discontinuities and intermittency statistics. Comparison of ACE solar wind data and simulations of 2D and 3D turbulence shows good agreement in waiting-time analysis of magnetic discontinuities, and in the related distribution of magnetic field increments. This supports the idea that the magnetic structures in the solar wind may emerge fast and locally from nonlinear dynamics that can be understood in the framework of nonlinear MHD theory. The analysis suggests that small scale current sheets form spontaneously and rapidly enough that some of the observed solar wind discontinuities may be locally generated, representing boundaries between interacting flux tubes.
\end{abstract}

\section{BACKGROUND}

A well known feature of solar wind observations is the appearance of sudden changes in the magnetic field vector. These changes are mainly directional, not in magnitude, and are detected throughout the heliosphere $[3,21,22,27]$. Changes are often seen at time scales of 3 to 5 minutes, although similar discontinuities are seen at smaller time increments [28]. A familiar interpretation is that these are classical ideal magnetohydrodynamic (MHD) discontinuities [3, 22, 27], a view consistent with the interpretation that other MHD scale fluctuations are noninteracting Alfvén waves. An alternative viewpoint is that both the fluctuations and discontinuities are facets of a nonlinear MHD cascade [1,23], and that these are interacting, not passive, and contribute, e.g., to heating of the interplanetary plasma. In the former view the interplanetary medium evolves very little, and its features can be traced back to features in the lower corona, possibly even to the photosphere [2]. Here we review and discuss observational and theoretical issues related to interplanetary discontinuities, making comparisons with moderate to high Reynolds number MHD simulations. We find that methods for identifying classical discontinuities and for computing quantities related to intermittency are closely related. These approaches give very similar results when used as a basis for identifying "events" in either simulation data or in ACE solar wind magnetic field data. In the simulations, we find that the typical events are connected with current sheets that form between adjacent magnetic flux tubes. Indeed this is consistent with the fact that the solar wind exhibits many properties associated with intermittent turbulence $[4,6,16,17,25]$, but the question persists as to whether these properties arise locally or if they are remnants of coronal processes (e.g., [2]). Here we will argue that coherent structures and therefore discontinuities can arise rapidly, and we suggest that at least some of the observed interplanetary discontinuities are formed locally.

\section{CELLULARIZATION, TURBULENCE AND DISCONTINUITIES}

The presence of discontinuities in the observed interplanetary magnetic field is suggestive of some kind of internal boundaries in the plasma. But there are other indications as well. A particularly intriguing example is the phenomenon of "dropouts" in solar energetic particle observations. In these sporadic events the intensity of energetic particles suddenly turns off, and on again, sometimes repeatedly within a few hours of data[19]. These observations may appear to be at odds with estimates of rates of perpendicular diffusion of energetic particles, but it transpires that considerations of the structure of the turbulent coronal and interplanetary magnetic field

CP1216, Twelfth International Solar Wind Conference,

edited by M. Maksimovic, K. Issautier, N. Meyer-Vernet, M. Moncuquet, and F. Pantellini

(C) 2010 American Institute of Physics 978-0-7354-0759-6/10/\$30.00 
$[11,12]$ can reconcile the observations with ideas of particle diffusion. In particular [11] turbulent magnetic flux tubes that occur in highly anisotropic (nearly 2D) turbulence models can provide trapping centers that prevent some magnetic field lines, those near strong magnetic O-points, from escaping. Particles with gyrocenters on these field lines may experience a delayed escape, producing steep gradients and a delay in attaining asymptotic diffusive transport. In this view, diagnostics that describe SEP dropouts [19] are providing us with pictures of the cellularized interplanetary magnetic field.

Here we will examine the occurrence and generation of internal magnetic boundaries. The main diagnostics we examine will describe properties of the magnetic field $\mathbf{B}=\mathbf{B}_{\mathbf{0}}+\mathbf{b}$ which is assumed to consist of a mean part $\mathbf{B}_{0}$ and a fluctuation $\mathbf{b}$. The former may vary slowly in space while b is a complex turbulent field that varies in space and time. To describe rapid changes in the magnetic field, we look at the increments $\Delta \mathbf{B}_{s}=\mathbf{B}(s+\Delta s)-\mathbf{B}(s)$ at points in space separated by $\Delta s$ along some trajectory. When $s$ is an inertial range separation, the increments have properties characteristic of the inertial range of turbulence[20]. A slightly more economical description, and one that relates well to discontinuity analysis is obtained by looking at the time series and statistics of the magnitude of the vector increments,

$$
|\Delta \mathbf{B}|=|\mathbf{B}(s+\Delta s)-\mathbf{B}(s)|
$$

again separated by $\Delta s$ and we now suppress the argument $s$ where convenient.

In Fig. 1 we show two samples of time series of $|\Delta B|$, one obtained from a 3D spectral method simulation of MHD by sampling along diagonals and another obtained from interplanetary magnetic field data measured by the ACE spacecraft $[13,14]$. Comparable numbers of correlation scales are shown for each. It is apparent that both datasets are spiky, and the events that might be identified as discontinuities are evident. While discontinuities are sometimes picked out using more elaborate methods (e.g., [28]), the baseline property that there is a large sudden change of direction, can be associated with a simple cutoff or threshold applied to the datasets shown in the Figure.

In the case of the simulation data it is possible to unambiguously identify what structures are associated with these discontinuity "events." This is particularly straightforward in two dimensions (2D), as illustrated in Fig. 2. This illustrates field lines and intensity of electric current density for a 2D incompressible MHD simulation of fully developed turbulence. It is a decaying turbulence run at moderate Reynolds number ( a few thousand) carried out with a very accurate and well resolved $512^{2}$ Fourier pseudospectral code. The picture, showing the system after just a few eddy turnover times, displays many magnetic
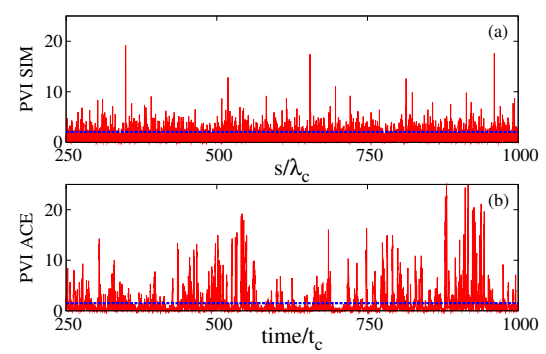

FIGURE 1. (color online) Time/space series of the magnitude of magnetic vector increments computed from solar wind ACE data and MHD simulation. In both cases data is acquired along a linear path (in solar wind using frozen-in flow) and normalized to the respective correlation scales. Here the scales are roughly comparable in terms of correlation scales, and the appearance of the datasets is similar, with spiky changes seen in both cases.

islands, some of which are reconnecting with a nearest neighbor. Many of the islands are also bordered by strong sheets of electric current density. When these are sampled by crossing them, the result appears as a tangential discontinuity (TD) for inertial range increments. (Note that the current sheets thicknesses are at around the dissipation scale.) None of these features were present in the initial data (not shown) in which the electric current is not concentrated but rather is randomly distributed by construction.

This observation of the generation of current sheets and their connection to TDs that can be "observed" in simulations leads naturally to the question of whether there might be a similar origin of discontinuities in solar wind turbulence. This question has been examined in some detail [13] by looking at the distribution of waiting times between discontinuity events identified either by classical methods (designated "TS") or by a threshold on the value of $|\Delta \mathbf{B}|$ normalized to its own variance (designated PVI"). The latter quantity is related to statistics that describe intermittency in turbulence. Greco et al found that the two methods performed almost interchangeably - most of the same events were identified and the waiting time distributions were almost identical.

Next Greco et al [14] applied these method to compare statistics of simulations and statistics of solar wind ACE magnetic field data. Use of a normalization of the increments to the variance $[7,25]$ facilitates and clarifies these comparisons. It transpires that the normalized waiting time distributions between events (using either TS or PVI) were extremely similar in the solar wind and simulation datasets at (inertial range) separations shorter than the correlation scale. At larger separations the distributions differ. 


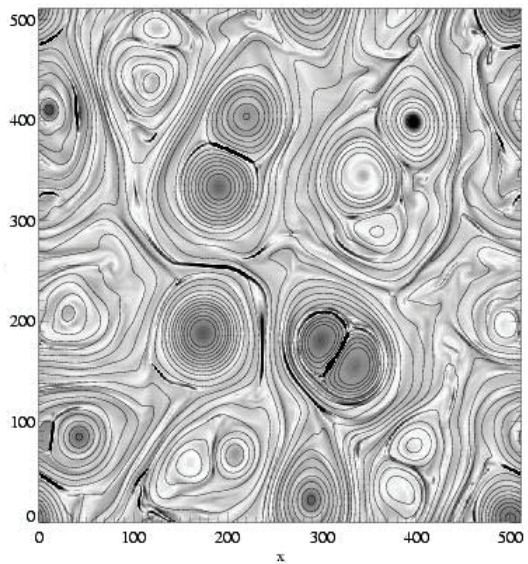

FIGURE 2. A contour map of field lines with superposed gray-shade plot of electric current density, from 2D MHD simulation. Strong current regions (very dark and very light regions) are frequently found between adjacent magnetic flux tubes, inducing a cellular structure. This feature is very clear in $2 \mathrm{D}$ and is present but more complex in $3 \mathrm{D}$ simulations. These current sheets appear as tangential discontinuities in the data samples, as in Fig. 1. It seems reasonable to suppose that a similar cellularization and origin of discontinuities might take place in the solar wind. Single spacecraft data cannot provide an unambiguous direct answer to this question.

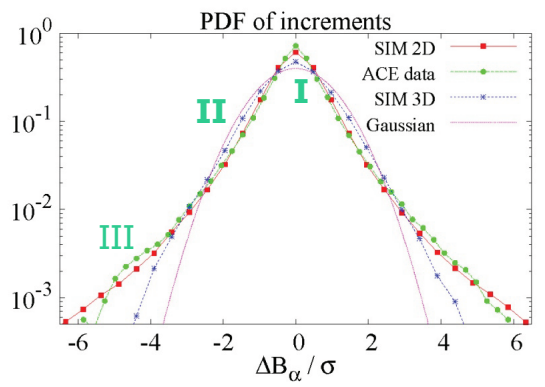

FIGURE 3. (color online) Probability distribution of magnetic increments from ACE data and both 2D and 3D simulations. Labels designate (I) super-Gaussian core, (II) subGaussian wings and (III) super-Gaussian tails.

\section{NON-GAUSSIAN STATISTICS}

The same study [14] also found that probability distributions (PDFs) of the normalized (component) increments also compare well. See Fig. 3, which illustrates PDFs of inertial range increments from $2 \mathrm{D}$ and $3 \mathrm{D}$ simulations, and ACE magnetic field data. A crucial point to realize is that the events that are identified as discontinuity events in either TS or PVI methods are associated with the tails of these distributions. The only technical difference is that PVI employs a threshold on the magnitude $|\Delta \mathbf{B}|$ while the PDFs shown here are those of a carte-

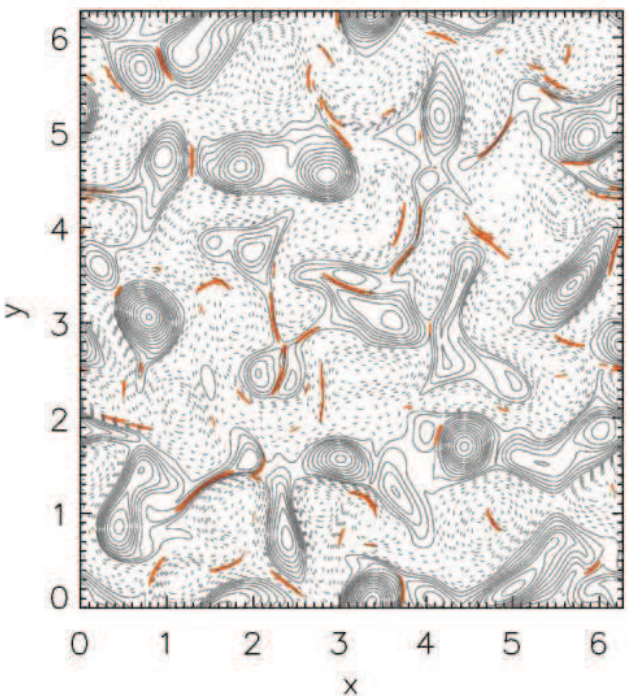

FIGURE 4. (color online) Magnetic islands from 2D simulation with region III, the super-Gaussian tails, shaded in. The shaded regions correspond to the current sheets between flux tubes, as in Fig. 1.

sian component component $\Delta B_{\alpha}$. The distributions are remarkably similar, with cross-overs between sub- and super-Gaussian regions occurring at very nearly the same value of normalized separation.

In Fig. 3 we also suggest a classification into (I) superGaussian core, (II) sub-Gaussian wings at intermediate values, and (III) super-Gaussian tails. We can then ask the question: where are the super-Gaussian tails coming from? In the case of the 2D simulations this is readily addressed by masking region III and visualizing the results. Fig. 4 shows that Region III is due to the current sheets that form between the flux tubes. These current sheets represent the well-known small scale coherent structures of MHD turbulence $[8,18,24,29]$ that are linked to the magnetic field intermittency.

The issue of whether discontinuities and current sheets can form due to turbulence in the solar wind depends on whether sufficient time passes for their formation. In the simulations described above the MHD system was examined after a few nonlinear times, beginning from a random phase initial condition with no coherent structures. For the solar wind case, one may easily estimate the number of nonlinear times (or age) in passing to $1 \mathrm{AU}$. We can base this on a fluctuation strength of $v=10-20 \mathrm{~km} / \mathrm{s}$, a correlation length of $L=0.01 \mathrm{AU}$, and an average solar wind speed of $V_{s w}=400 \mathrm{~km} / \mathrm{s}$. A simple nonlinear time scale is $L / v$ and the transit time to position $R$ in the heliosphere is $R / V_{s w}$. Therefore the age of the turbulence at 1 $\mathrm{AU}$ is about $(v / L)\left(R / V_{s w}\right) \approx 2$ to 4 nonlinear times. Even though this is probably somewhat of an overestimate due to effects of Alfvénicity, it appears that the turbulence has 
time to become fully developed in transit to $1 \mathrm{AU}$. This is a familiar conclusion, for example due to the frequent observation of features such as Kolmogoroff-like power spectra. However recent studies make it clear that some features of turbulent relaxation occur locally, on the scale of typical flux tubes, and rapidly, on the time scale of a fraction of a global nonlinear time scale[24]. In particular the formation of strong boundaries of current sheets between flux tubes seems to be a rapid process.

To make this point even more strongly, we note that another recent study [30] finds that non-Gaussian statistics emerge very rapidly from band -limited initial conditions, and that this process is essentially ideal. Therefore the effects that drive the formation of the characteristic coherent structures (i.e., the current sheets) are ideal, nonlinear and very rapid. The key processes that can generate discontinuities and flux tube boundaries can act in less than a nonlinear time. This means that in transit to $1 \mathrm{AU}$ the solar wind should generate non-Gaussian features, including current sheets.

\section{CONCLUSIONS}

We can draw a firm conclusion for the numerical experiments, that the discontinuity events are formed spontaneously due to nonlinear couplings, cascade and turbulence. They were not present in the initial data. The extension of this conclusion to the solar wind is tempting, but remains uncertain.

It seems likely that in 3D solar wind turbulence, intermittency and the associated coherent structures and current sheets will have time to form. One might conclude that, while some features related to interplanetary magnetic boundaries may survive in transit over $1 \mathrm{AU}$ [2], at least some of these features are probably generated by in situ processes. However the mix of remnant TD/current sheets and turbulence-generated TDs/current sheets remains an incompletely understood question. It remains of importance however, in understanding the near-earth solar connection, predictability in space weather, solar energetic particles, and other observational features of the Geospace environment.

Finally we note that coherent structures and intermittency of the type that we analyzed here can also be investigated using more advanced techniques, such as those related to multifractal analysis [9], and direct measures of phase coherence $[10,15]$.

This research supported in part by the NSF SHINE (ATM0752135) and solar-terrestrial (ATM0539995) programs, and by the NASA Heliophysics Theory Program (NNX08AI47G).

\section{REFERENCES}

1. Biskamp, D., and W.-C. Muller, Phys. Plasmas, 7, 4889 (2000).

2. Borovsky, J., J. Geophys Res. 113, A08110, 2007JA012684 (2008).

3. Burlaga, L. F., Solar Physics, 4, 67 (1968).

4. Burlaga, L. F., J. Geophys. Res., 96, 5847-5851 (1991).

5. Burlaga, L. F., C. Wang, J.D. Richardson, and N.F. Ness, Astrophys. J., 585, 1158 (2006).

6. Burlaga, L. F., N. F. Ness and M.H. Acuna, J. Geophys. Res., 111, A09112 (2006).

7. Bruno, R., Carbone, V., Veltri, P., Pietropaolo, E., Bavassano, B. Plan. Space Sci., 49, 1201 (2001).

8. Carbone, V., P. Veltri,and A. Mangeney, Phys. Fluids A, 2, 1487 (1990).

9. Chang, T. and C-C. Wu, Phys. Rev. E, 77, 045401 (2008).

10. A. C.-L. Chian and R. A. Miranda, Ann. Geophys. 27, 1789 (2009).

11. Chuychai, P., D. Ruffolo, W. H. Matthaeus and G. Rowlands, Astrophys. J., 633, L49 (2005).

12. Giacalone, J., J. R. Jokipii and J. E. Mazur, Astrophys. J., 532 L75 (2000).

13. Greco, A., Chuychai, P., Matthaeus, W. H., Servidio, S., Dmitruk, P., Geophys. Res. Lett. 35, L19111, 2008GL035454 (2008).

14. Greco, A., W. H. Matthaeus, S. Servidio, P. Chuychai, and P. Dmitruk, Astrophys. J., 691, L111 (2009).

15. Hada, T., Koga, D., and Yamamoto, E., Space Sci. Rev., 107, 463, (2003).

16. Horbury T. A., Balogh A., Forsyth R. J., and Smith E. J., Adv. Space Phys. 19, 847 (1997).

17. Marsch, E., Tu, C. Y., Annales Geophysicae, 12, 1127 (1994).

18. Matthaeus, W. H., Lamkin, S. L., Physics of Fluids, 29, 2513 (1986)

19. Mazur, J. E., G. M. Mason, J. R. Dwyer, J. Giacalone, J. R. Jokipii, and E. C. Stone, Astrophys. J., 532, L79 (2000).

20. Monin, A. S., Yaglom, A . M., Statistical Fluid Mechanics (MIT Press, Cambridge, MA), 2 (1975)

21. Neugebauer, M., J. Geophys. Res., 111, 4103 (2006).

22. Ness, N. F. and L. F. Burlaga, J. Geophys. Res., 106, 15803-15817 (2001).

23. Politano H., Pouquet A., and Carbone V., Europhys. Lett. 43, 516 (1998).

24. Servidio, S., Matthaeus, W. H., Dmitruk, P., Physical Review Letters, 100, 095005 (2008).

25. Sorriso-Valvo, L., Carbone, V., Veltri, P., Consolini, G., Bruno, R., Geophys. Res. Lett., 26, 1801 (1999).

26. Sorriso-Valvo, L., Carbone, V., Veltri, P., Politano, H., Pouquet, A., Europhysics Letters, 51, 520 (2000).

27. Tsurutani, B. T., Smith, E. J., J. Geophys. Res., 84, 2773 (1979).

28. Vasquez, B. J., Abramenko, V. I., Haggerty, D. K., Smith, C. W., Journal of Geophysical Research (Space Physics), 112, 11102 (2007).

29. Veltri, P., Plasma Phys. Contr. Fusion, 41, 787-795 (1999).

30. Wan, M., S. Oughton, S. Servidio, and W. H. Matthaeus, Phys. Plasmas, 16, 080703 (2009). 